Estudios sobre armas antiguas, arte militar y vida cultural en oriente y occidente XXX (2010), pp. 99-116 ISSN : 0436-029X

doi: $10.3989 /$ gladius.2010.0005

\title{
RESTOS DEL ARMAMENTO DE LA LEGIO IIII MACEDONICA HALLADOS EN SU CAMPAMENTO DE HERRERA DE PISUERGA (PALENCIA, ESPAÑA)
}

\author{
REMAINS OF WEAPONS AND ARM OUR OF LEGIO IIII MACEDONICA FOUND IN ITS \\ CAMPAT HERRERA DE PISUERGA (PALENCIA, SPAIN)
}

POR

Carmelo Fernández Ibáñez

\section{Resumen - A bstract - Résumé}

A partir de cuatro áreas de excavación Ilevadas a cabo en la población de Herrera de Pisuerga (Palencia) cuyo potencial estratigráfico entre otras ocupaciones aportó restos de la legio IIII M acedonica, se muestran, comentan y estudian los diferentes tipos de armas recuperadas y que pertenecieron a esta unidad del ejército de Roma que participó en la conquista del norte de H ispania, y que allí permaneció entre los años 20/15 a.C.-40 d.C.

Four areas, excavated at the town of Herrera de Pisuerga (Palencia), have revealed evidence of different occupations in their stratigraphic sections, including remains of the Legio IIII Macedonica. This paper describes, discusses and studies the different types of weapons that have been found. They bel onged to this unit of the Roman army, which took part in the conquest of northern Hispania and which remained in the town between 20/15 BC and $40 \mathrm{AD}$.

Les fouilles du site d'Herrera de Pisuerga (Palencia) ont permis de mettre au jour, dans quatre secteurs, différents niveaux d'occupation, dont I'un a livré des témoignages de la legio IIII M acedonica. Cet article décrit et discute les différents types d' armes retrouvées que l'on peut attribuer à cette unité de l' armée romaine ayant partici pé à la conquête de l'Hispanie septentrionale puis demeurée sur place entre 20/15 a.C. et 40 p.C.

Palabras Clave - Key words - Mots clés

Altoimperial; M ilitaria; M etalistería; Pisoraca; A rmas.

Early Empire; Militaria; M etal-working; Pisoraca; Weapons.

Haut-Empire; M ilitaria; M étallurgie; Pisoraca; Armes.

\section{INTRODUCCIÓN}

La legio IIII Macedonica fue un cuerpo del ejército de Roma que, ante el silencio de las fuentes literarias, los datos arqueológicos parecen demostrar que entró en combate en la última fase de la conquista del norte de Hispania, y sobre todo en la pacificación de toda aquella área (M orillo Cerdán, 2000: 610). A las órdenes de M. V. A grippa actuó contra las poblaciones

* M useo de Palencia, Plaza del Cordón no1, 34001 Palencia (España) Correo electrónico: feribaca@jcyl.es 
indígenas de los Cantabri al final de la contienda, estableciendo su base campamental en el área que hoy ocupa una población del N.E. de la provincia de Palencia: Herrera de Pisuerga. Desde 1983 y hasta la actualidad se van Ilevando a cabo trabajos de campo al ritmo de las necesidades urbanísticas y de investigación. Dentro el material exhumado en las campañas de excavación 1983-1990 nuestra responsabilidad fue el estudio de los objetos de metal de los diferentes cuerpos de ejército allí asentados (Fernández Ibáñez, 1992; 2002a). EI material bélico que presentamos es el que pertenece a la legión responsable de la primera ocupación romana, y por lo tanto es parte de aquel estudio.

\section{EL YACIMIENTO Y LA HISTORIOGRAFÍA DE SU INVESTIGACIÓN}

Bajo la actual población de Herrera de Pisuerga, localidad situada al noreste de la provincia de Pal encia (fig. 1) se encuentran ocultos importantes restos de época romana, cuyas investigaciones demuestran que aquel lugar tuvo en la antigüedad una tan azarosa como históricamente interesante vida, mucha de la cual tuvo carácter logístico-militar. Las poblaciones que allí se asentaron lo hicieron sobre un cerro elevado respecto al terreno circundante, cercano en la antigüedad a la confluencia de los ríos Pisuerga y B urejo (uno de los afluentes de aquél). Sobre los restos de una población quizás de la la E dad del Hierro hoy escasamente conocidos, hacia el año 20/15 a.C..$^{1}$ y tal vez ya concluida la guerra -o bien muy cerca de su conclusióncontra las poblaciones indígenas de Cántabros y A stures, la legio IIII M acedonica levantó un primer campamento (que se remodeló en época de Tiberio) para llevar a cabo la explotación, organización y control sobre el territorio recién conquistado. Tras abandonarlo hacia el año 40 d.C. para asentarse en el área donde se levanta la actual ciudad de M ainz (M ogontiacum), el ya viejo emplazamiento en $\mathrm{H}$ ispania se transformó en castellum bajo el reinado de Claudio o Nerón con la presencia del Ala Parthorum. Esta tropa equitata permaneció hasta finales de este siglo I o principios del siglo II d.C., cuando fue sustituida por la Cohors I Gallica (Fernández Ibáñez, 2001) (¿o la I Celtiberorum?) (M orillo Cerdán y Fernández Ibáñez, 2001-02) hasta aproximadamente la mitad de este último siglo. Posteriormente en la historia fue núcleo militar visigodo y en la Edad M edia se erigió un castillo hoy desaparecido junto al núcleo poblacional anejo (Pérez González y A rroyo Rodríguez, 2003: 48-65).

Tras las primeras excavaciones Ilevadas a efecto en 1932 por J. M artínez Santa-Olalla en la necrópolis visigoda (M artínez Santa-Olalla, 1933), no fue hasta 1960-61 cuando A. García y B ellido retoma las investigaciones pero esta vez en busca de la aclaración de los asentamientos de época romana (García y B ellido et alii, 1962; 1970: 3-24)². Posteriormente en 1976 un equipo de la U niversidad de Valladolid dirigido por A. B alil y J. A. A básolo excava el área de La Chorquilla. Desde 1983 y hasta hoy C. Pérez y E. Illarregui de la U.E. de Segovia junto a un equipo de especialistas han sido los encargados de las excavaciones y el estudio de los materiales de entre los cuales aquí destacamos los objetos de metal (Fernández I báñez, 1992; 1999b; 2002a; 2004a; 2006a), al ritmo de las necesidades urbanísticas del actual núcleo poblacional y su territorio inmediato.

\footnotetext{
1 A Igunos objetos cerámicos aún no publicados parecen retrotraer esta fecha hasta el 25/20 a.C.: M orillo Cerdán, Pérez González e Illarregui, 2006: 316.

2 El estudio de los objetos metálicos procedentes de estas excavaciones han sido publicados hace escasos años (Fernández Ibáñez, 2006a).
} 


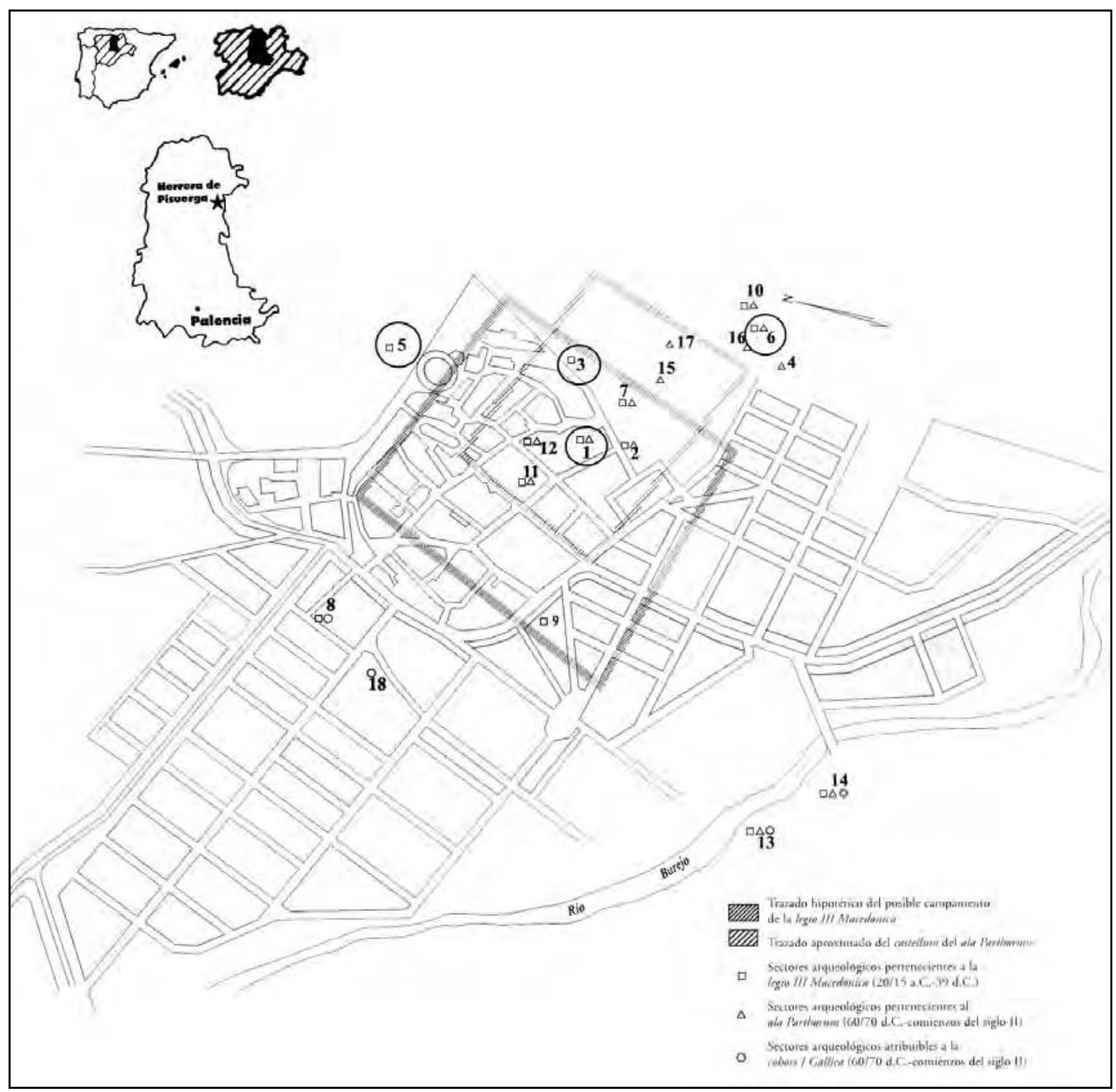

Figura 1. Situación geográfica de la población de Herrera de Pisuerga. Sobre el plano de la localidad, los números indican las áreas más importantes donde se han llevado a cabo excavaciones. A quellas que se encuentran encerradas por círculos, se corresponden con los lugares de donde proceden los objetos tratados en este estudio: (1) Cuartel I, (3) La Chorquilla, (5) El Castillo y (6) San M illán. Los sectores paralelepípedos superpuestos, indican la extensión aproximada de los campamentos militares; el de mayores dimensiones castra legionis IIII Macedonicae, y el más reducido el castellum para tropas de auxiliares (según M orillo, Pérez e Illarregui, ligeramente modificado por el autor).

\section{LAS ÁREAS ARQUEOLÓGICAS}

En función de como hoy en día se conciben buena parte de las intervenciones arqueológicas que en cualquier yacimiento se llevan a efecto de forma continuada, y sobretodo, en función de las ineludibles necesidades contemporáneas de expansión y remodelación que genera un núcleo poblacional que encierra en su subsuel o un rico patrimonio arqueológico, en Herrera de Pisuerga las excavaciones practicadas en los últimos años -mayoritariamente de tipo pre- 
ventivo y de urgencia- dentro del casco histórico y su perímetro aledaño, son producto de las necesidades urbanísticas o de tipo más o menos similar. Por lo tanto, hasta ahora la planificación de las intervenciones ha venido de la mano de una tan necesaria como urgente actuación conservativa, caracterizada por la aleatoriedad que las exigencias imponían. M ás no por ello ha dejado de tener importancia esta metodología así como los datos que han ido obteniéndose en cada lugar excavado. Para ordenar dichas excavaciones en todo tiempo, desde un principio se dividió el casco urbano en cuatro grandes áreas o «sectores» (A - D), más otros dos al Sur y Norte (E y F): son el río B urejo y la carretera Nacional 611 (Santander-Palencia) como hitos topográficos más destacables.

Cuatro han sido los lugares o zonas donde las pertinentes excavaciones han llegado a aportar documentación material en sus niveles más inferiores, relacionadas todas ellas con la fundación del campamento y por lo tanto con la legio III M acedonica. Todas estas áreas se reagrupan en el ángulo N.E. de la actual población, y en los cuadrantes B (sobretodo) y C de aquella subdivisión inicial. Tales áreas son (fig. 1):

- Cuartel I: Entre 1983-86 se excavó el patio del antiguo cuartel de la Guardia Civil, lugar situado al S.E. de la población y dentro del sector «C». Pudieron distinguirse cuatro niveles de habitación y, del estudio del material cerámico (Pérez González, 1989), lucernas (M orillo Cerdán, 1999), numismático (M orillo Cerdán y Gómez Barreiro, 2006) y metálico (Fernández Ibáñez y Pérez González, 1990; Fernández Ibáñez, 2004a) del nivel más profundo o fundacional (nivel IV), se pudo determinar que abarca toda la vida campamental de la legio IIII M acedonica, o sea, entre el 15/20 a.C. y el 40 d.C.

- San M illán: Se trata de un gran vertedero situado al S.E. de la población y dentro del sector «B». Fue excavado ente 1989-90 proporcionando en cinco niveles una muy interesante y completa información de las estancias campamentales de Herrera de Pisuerga, además de abundante y muy variado material. El último (nivel V) fue subdividido en otros dos, siendo de nuevo el anális de las monedas y lucernas las que nos aportan cronologías acordes con la presencia de la legión de nuestro interés (M orillo Cerdán, 1999; M orillo Cerdán y Gómez Barreiro, 2006): Va (el superior: 10 a.C.-40 d.C.) y V b (10 a.C.-10 d.C.).

- La Chorquilla: Es un término emplazado al N.E. de la población de Herrera de Pisuerga $y$ dentro del sector « $B$ », en la misma margen derecha del río Pisuerga y que hasta él desciende en suave declive. Se trata de una gran área de vertido de deshechos entre cuyos hallazgos lo más destacable es el gran volumen de hallazgos de cerámica sigillata, descollando por su calidad las producciones itálicas. Son muy escasos los objetos de metal. Sobre un suelo natural de carácter arcilloso que se alcanza a más de cuatro metros de profundidad, las más recientes excavaciones llevas a cabo en 1988 y 1991-92 pusieron al descubierto siete niveles. Tras el estudio del material numismático y las lucernas los estratos más profundos y que aquí nos interesan ofrecieron las siguientes cronologías: Nivel IV (10 a.C.-10 d.C.), Nivel V (10 a.C.10 d.C.), Nivel V/VI (20 a.C.-0), Nivel VI/NII (20 a.C.-0) (M orillo Cerdán, 1999; M orillo Cerdán y Gómez B arreiro, 2006; Pérez González, 1989).

- El Castillo: El extremo noreste de la población (sector «B») es el límite de una antigua terraza el evada sobre el territorio circundante donde hasta los años cuarenta del pasado siglo XX se levantaba un castillo medieval. En su ladera norte y entre los años 1986-88 se practicaron excavaciones que dieron como resultado un vertedero militar con una potencia media de más de tres metros. El yacimiento mostraba seis niveles (con subdivisiones algunos de ellos), de los cuales el quinto y siempre según los estudios de numismática y lucernas (M orillo Cerdán, 1999; M orillo Cerdán y Gómez Barreiro, 2006) es el que abarca el período que aquí 
nos interesa, y que muestra las siguientes cronologías: Va (10 a.C.-40 d.C.) y Vb (10 a.C.-10 d.C.).

\section{LAS ARMAS}

L as áreas arqueológicas anteriormente vistas han aportado un variado conjunto fundamentalmente de fragmentos de armas, aunque no están representadas la totalidad de las que actualmente se conocen en el ejército de Roma. Resultan especialmente escasas las propiamente defensivas por las cuales vamos a comenzar.

\section{- D efensivas}

\section{- Corazas}

Dentro del tan abundante número de pequeños objetos recuperados durante las diferentes campañas de excavación, ninguno con seguridad puede ser identificado con al guna de las partes de una lorica segmentata. A I igual podemos decir con respecto a la lorica hamata, excepto, quizás, un objeto con doble sección que creemos haber identificado como una de las dos partes ( $54^{\prime} 7 \mathrm{~mm}$ longitud) (fig. 2,1) en las que se encuentra conformado un fijador/sustentador de las solapas/hombreras de una coraza de malla3. El aspecto formal de este objeto moldeado en aleación de cobre puede suscitar dudas en cuanto a su clasificación. A unque en la generalidad estos objetos suelen estar muy decorados y tener aspecto liriforme, algunos ejemplos como los de Bagington o Camerton (Chew, 1991: 42) varían ligeramente la forma en «S» que es norma, creando nuevos quiebros curvos y por lo tanto estableciendo variantes. El objeto que aquí presentamos quizás procedente de Herrera de Pisuerga, y al ser un hallazgo de fortuna, no es posible asignarlo categóricamente a la legio M acedonica. A hora sí, es el primer objeto de estas características que se ha encontrado hasta hoy en $\mathrm{H}$ ispania y también la primera muestra de lorica hamata procedente del yacimiento de Herrera.

\section{- Ofensivas}

- Puñales y vainas

Dos empuñaduras de daga que presentan distinta tipología son los únicos hallazgos de este tipo de arma blanca. Son de hierro y proceden ambas de las antiguas excavaciones en el vertedero de La Chorquilla y por lo tanto sin claro contexto (Fernández Ibáñez, 2006a; García y Bellido et alii, 1970: 19, fig. 22), pidiéndolas considerar como objetos desechados en la antigüedad. La diferencia entre ambas radica esencialmente en el pomo, siendo una de tipo bidiscoidal ${ }^{4}$ (fig. 3,2) y la otra de empuñadura en «D» invertida (Fernández I báñez, 2008: 95) 0 «semidisco» (Kavanagh de Prado, 2008: 29-33)5 (fig. 3,1). Según los estudios más recientes

3 Objeto que forma parte de la pequeña colección «A lba» hoy custodiada en el M useo de Palencia. Su pequeño grupo de objetos metálicos romanos sin aparente procedencia y entre los que se encuentran varios de clara adscripción militar, cuenta con una información no contrastada la cual los atribuye a que tienen a Herrera de Pisuerga como procedencia.

4 Empleamos esta denominación, más correcta que aquella otra (biglobular) tan al ternativa como incorrecta, según una reciente propuesta razonada ( $\mathrm{K}$ avanagh de Prado, 2008: 6).

5 K abanav (2008: 39-40) incluye la empuñadura en «D»0 «semidisco» hallada en Herrera de Pisuerga, en lo que a arriaces se refiere, dentro del grupo «B» (arriaz «abatido») de su tipología, junto a los de Palencia capital (Fernández Ibáñez, 2004b: Fig. 1, 1 y 7, 8; 2006b: 285; 2007: 409-411; 2008: figs. 9, 10 y 16). Si bien tiene razón en lo que a esta ob- 
sobre las dagas romanas halladas en la Península Ibérica, la primera empuñadura se corresponde morfológicamente con el arma más antigua, ya que hereda su forma del último modelo indígena celtibérico (Fernández Ibáñez, 2008: 107-111) de donde procede como también ha determinado K avanagh (2008: 22-23, 31). Desde ejemplares tan antiguos fechados entorno a la década de los años cincuenta a.C. (R aso de Candel eda, Á vila) ${ }^{6}$, a este de Herrera de Pisuerga le hemos propuesto como uno de los hallazgos que muestran los últimos ejemplares de tipología bidiscoidal. Que si bien no ha sido posible fecharlos debido a que al ser hallazgos recuperados hace ya años (al igual que el de Numancia), pensamos que el final de su uso se encuentra entorno al cambio de era, aunque por el momento no contamos con argumentos determinantes. Quizás también y como veremos para con los contingentes de arqueros o mismamente honderos, pueden ser algunas de las evidencias materiales que nos muestren determinados cambios producidos cuando A ugusto alcanza la púrpura imperial.

En lo que a vainas y fundas se refiere hemos identificado su presencia tan solo por varios pequeños clavillos con cabeza (con punto en relieve en el centro de esta) de sección troncocónica o recto-convexa (fig. 2,4 ) para unir ambas láminas de anverso y reverso. También, una presilla o gozne moldurada (fig. 2,2) para la inserción de anillas de suspensión, y varias de estas últimas (entre 19 y $21 \mathrm{~mm} \varnothing$ ) con secciones circular, oval o triangular ( $\varnothing 13^{\prime} 5 \mathrm{~mm}$ ). Todo este conjunto fabricado en aleación de cobre procede del nivel V de San M illán.

\section{- Espadas y vainas}

Como es posible apreciar hasta ahora, la armamentística que mostramos mayormente se basa en el reconocimiento y estudio de pequeños fragmentos, escasos en número, pero representativos de cada tipo de arma. Es pura y auténtica investigación basada en la reconstrucción de un todo a partir de pequeñas partes; esto mismo es posible aplicar a los restos de gladii y sus vainas. Todas las evidencias que mostramos se encuentran fabricadas en al eación de cobre y proceden del nivel $\mathrm{V}$ del área de San M illán. Del arma propiamente dicha solo una cazoleta hemiesférica ( $50 \mathrm{~mm} \varnothing$ y $122 \mathrm{~mm}$ de altura) conformada en fina chapa ( $1 \mathrm{~mm}$ de espesor) que se corresponde con parte de un pomo (fig. 2,5). Es difícil establecer de que tipo de arma se trataba (probablemente model os precoces como M ayenza o Pompeya) pues ambos model os utilizaban pomos semiesféricos - $u$ oblongos-, bien macizos en hueso, 0 en metal como es este caso y que seguramente recubrían un alma de materia orgánica (¿madera?) a la cual permanecían unidos. L as dos semiesferas metálicas y su núcleo eran atravesados por el extremo proximal de la espada que era una fina barra destinada también al emplazamiento del arriaz y la caña de la empuñadura, permaneciendo unido este conjunto por la presión ejercida entre las diferentes partes de la empuñadura. Q ue remataba en un pivote también metálico en el extremo proximal del arma, y que el pomo procedente de Herrera de Pisuerga parece que no tenía.

\footnotetext{
servación se refiere según se desprende de las ilustraciones publicadas por nosotros, estas serían armas que más bien fuese necesario incluir dentro del grupo «C» (arriaz «en T invertida») de entre los que dicho autor propone. Esta observación nuestra viene abalada al haber sido nosotros los que hemos proporcionado a dichas piezas el tratamiento de conservación y restauración que precisaban con vistas a su más correcto análisis histórico y conservación al futuro. El precario estado de conservación que les es característico a prácticamente la inmensa totalidad los antiguos objetos de hierro hallados en el norte de la Península Ibérica, juega malas pasadas a los investigadores, que siempre han de tenerlo muy en cuenta de cara a las observaciones que realicen en lo que los aspectos formales de sus investigaciones se refiere, tal y como hemos repetido más de una ocasión (F ernández I báñez, en prensa). Tanto en objetos tratados como sobretodo y con más razón en los que aún no lo han sido y por lo tanto aún no han recibido la primera fase de limpieza. En el caso de los restos de las dagas antedichas, esta carencia se debe también a nuestra pluma el no haberlo indicado.

6 K avanagh (2008: 27) propone asimismo la daga hallada en el poblado ibérico de EI M olón (Valencia) (A ImagroGorbea et alii, 1996).
} 
En lo que a las vainas o fundas de estas armas se refiere, los restos conservados son de tres partes diferentes; proceden todos ellos nuevamente del nivel V de San M illán y se encuentran fabricados en cobre y/o aleación de este metal. Primeramente dos restos de las rejillas que decoraban la parte superior del anverso junto a la embocadura. Una de ellas se encuentra constituida por una celosía de laminitas entrecruzadas (fig. 2,6), y la otra un el emento circular compuesto por brazos curvados en número de siete y con sentido dextrógiro (fig. 2,7). Otros elementos son fragmentos de los dos característicos zunchos o láminas molduradas (fig. 2,8) que abrazaban y aprisionaban el tercio superior de la vaina, y de cuyos extremos colgaban las anillas que hacían suspender al arma de su tahalín. Dichas molduras son las que le confieren resistencia a este sistema de sustentación. Dentro de este aspecto se englobarían los últimos objetos que nos faltan por comentar, como son las anillas a las que antes hacíamos alusión (fig. 2,9 a 12). Hemos considerado que estas son robustas. M ás y de mayor diámetro que las utilizadas para la suspensión de las dagas; pero menos que otras con las que estas se suelen confundir, como son, las empleadas en el arnés equino para la distribución de riendas. Con diámetros en torno a los $20 \mathrm{~mm}\left(19^{\prime} 5,21,21 \mathrm{~mm}\right)$ presentan la particularidad que la sección es triangular (buscando la resistencia que en el metal proporcionan los quiebros y las aristas), bien sean macizas o huecas. No obstante también pensamos que pueden haber sido fabricadas con secciones circulares o en óvalo, ya sea desde su confección o bien como reparación.

Para finalizar este apartado solo nos queda hacer alusión a los llamados botones en ángulo, prendedores o abrochadores con botón y anillo (button loop, crochettes, knebelverschlüsse) que en base a una variada tipología, el volumen de hallazgos crece constante aumento habiendo ya superado la primigenia clasificación (Wild, 1970). A parecen de forma tan repetitiva como abundante en los emplazamientos militares, y aunque no de forma exclusiva sí en cantidades ostensiblemente más reducidas en hábitats civiles. D esde aproximadamente el siglo II a.C. al III d.C. (J ames, 2004: 82-84) (posiblemente incluso más), su simplicidad se basa en un «botón» que adopta diferentes formas y dimensiones (circular, cuadrangular, barra transversal plana o barrita circular, etc... ), y bajo el cual de desarrolla un apéndice en ángulo recto que finaliza mayoritariamente en un anillo (simple, doble, triple; circular, oval, triangular, rectangular, cuadrangular... ), que cosido quedaría unido a un el emento de tejido o bien de piel. Poseen en la generalidad reducidas dimensiones y normal mente se encuentran fabricados en aleación de cobre. Desde hace ya años se les ha venido atribuyendo una mera función de unión entre dos partes de una prenda (sagum o paenula) como botón o más específicamente corchete.

Oldenstein (1976-77: 186) observó que fundamental mente (aunque no con exclusividad) un determinado modelo de entre los descritos, que presenta, en vez del clásico botón circular una barrita transversal de sección plana o circular y anilla circular, o más comúnmente triangular, podía haber formado parte del tahalí para la sustentación de espadas y quizás también de dagas. Esta hipótesis ha ido tomando visos de verosimilitud, en función de los hallazgos efectuados en varios oppida de la Galia (Desbat y M aza, 2008: 240; Dechezleprêtre, 2008: 100; Pernet, Poux y Tejen, 2008: 114; Poux, Feugère y Demierre 2008: 216; Poux, 2008: 324326). Esencial mente este objeto serviría para unir los dos extremos de la correa de cuero que formaba parte del tahalí, y de la cual pendía la vaina de la espada, colgada del hombro derecho del soldado (Poux, 2008: 320).

7 El tahalí o sistema de unión entre la vaina y uno de los cinturones de donde se llevaría colgada en estos primeros tiempos, tal y como se observan en las representaciones de las estelas funerarias, no nos es conocido. En el nivel $V$ del área arqueológica de San M illán fue descubierta una doble placa de cinturón (hierro-bronce) que porta una gruesa anilla, que en su día interpretamos erróneamente como perteneciente al tahalí de una daga (Fernández Ibáñez, 1999b), pero qué duda cabe que su robusta envergadura la hacen más propia para un gladius. 
En Herrera de Pisuerga contamos con dos ejemplares de estos objetos que hemos detallado. U no de ellos de pequeña dimensión (23 mm de longitud) (fig. 2,3), si bien no resulta exactamente idéntico a lo que hemos descrito. El botón superior es una laminita rectangular y el ojal es circular. El otro ejemplar está fracturado y presenta un precario estado de conservación. A mbos proceden del nivel $V$ del área arqueológica de San M illán.

- A rmas arrojadizas

\section{Hastae y jabalinas}

L os hallazgos en este yacimiento muestran que las llamadas «puntas de lanza» (moharras) pueden agruparse en dos tipos en función de su tamaño. Con respecto al de mayores dimensiones solo dos objetos han sido identificados en hierro (solo uno de los ejemplares está completo y posee una longitud de $311 \mathrm{~mm}$ ), presentando ambas hojas lanceoladas de sección lenticular, y cónico el sistema de unión al hasta de madera. Proceden: de Cuartel I (nivel IV) el ejemplar completo habiendo sido hallado junto a un denario de la ceca Bolskan ${ }^{8}$ (Fernández Ibáñez, 2004a: 240, 247, 258) (fig. 3,3) ${ }^{9}$, y un fragmento de hoja de San M illán (nivel V).

En lo que concierne a las moharras con dimensiones más reducidas, en el nivel $V$ de San M illán se halló un pequeño conjunto de puntas que por su pequeño tamaño podemos considerarlas como pertenecientes a venablos: las completas muestran longitudes entre $92 \mathrm{~mm}$ y 125 $\mathrm{mm}$. Se encuentran forjadas también en hierro y presentan como las anteriores, características formales muy simples: hoja lanceolada (entre 55 y $100 \mathrm{~mm}$ de longitud) de sección lenticular o romboidal, y sistema cónico-rasgado de enmangamiento al hasta (entre $46-56 \mathrm{~mm}$ de longitud y $17^{\prime} 5-21 \mathrm{~mm}$ de $\varnothing$ ) (fig. 3,5 a 7). O tra hoja muy alterada fue recogida en el nivel IV de Cuartel I (fig. 3,4).

En lo que se refiere a los regatones estos se encuentran forjados en hierro y han sido hallados en todas las áreas arqueológicas aquí tratadas menos en L a Chorquilla. L os hay de tamaños y formas con ligeras variaciones ${ }^{10}$ (también fragmentados, cosa que suele ser corriente - fig. 4,3 ), aunque básicamente pueden agruparse en dos tipos. El primero y más sencillo a nivel formal es el ya bien conocido como cónico y siempre rasgado (fig. 4,2); solo se conoce un ejemplar con el extremo de sección cuadrada (fig. 4,4). Son los que poseen mayores dimensiones abarcando desde los $132 \mathrm{~mm}$ a los $56 \mathrm{~mm}$, y con embocaduras de diferentes diámetros: $13,19,21, \pm 25 \ldots \mathrm{mm}$. El segundo grupo hasta ahora lo forman dos pequeños ejemplares (uno de ellos completo de $66 \mathrm{~mm}$ longitud x $16 \mathrm{~mm} \varnothing$ procedente del nivel V de San M illán -fig. 4,1-; el otro, fragmentado, fue hallado en el nivel IV de Cuartel I) cuya característica adicional y que quizás más les identifica como grupo, es el poseer un extremo que no finaliza de forma aguda, sino que presenta un botón cónico o piramidal. Si bien no es un modelo tan usual como el anterior, sí parece ser bastante exclusivo de áreas militares romanas (Ham Hill, Hod

8 Tras el estudio de la numismática procedente de las diferentes ocupaciones militares en el área campamental de Herrera de Pisuerga, se ha concluido que el conjunto tardorrepublicano - todo él en plata- es numeroso y representativo, suponiendo una circulación residual dada la antigüedad de sus emisiones. Vinieron de la mano de los legionarios de la leg. IIII Macedonica que levantaron el primer campamento. M ás en concreto, la mayor parte de las monedas de la ceca Bolskan (tres de cuatro) corresponden a época sertoriana, habiendo servido para el pago de tropas en los diferentes años y desde aquella ya lejana guerra civil (M orillo Cerdán y Gómez B arreiro, 2006: 341-346).

9 Se trata de un ejemplar que en función de sus características formales son netamente indígenas, cuyos paral elos más cercanos los hallamos en poblados de la la y II a E dad del Hierro en la provincia de Navarra (Fernández Ibáñez, 2004: 247). Por lo tanto, quizás esta pieza se encuentra más relacionada con tropas de auxiliares.

10 Solo en un caso de entre todos los regatones es evidente la perforación para insertar el clavo-pasador que aseguraba al astil a estos extremos metálicos, aunque no se ha conservado. 
Hill, B rough-on-H umber, H ousesteads, L ongthope, Fisbourne, N ewstead, V indonissa, Petavonium... ) (Fernández Ibáñez, 2004a: 247-248; Feugère, 1993: 171); lógicamente también los hay cónicos de pequeño tamaño (fig. 4,2). A I menos teóricamente el peso del regatón debería de estar en cierta relación respecto a la moharra o punta, con el fin de que contrarrestarse y así lograr un mayor arco de tiro y por ende al canzar una distancia superior. Independientemente los regatones podrían tener relación con el tamaño de la punta, por lo que para hastae, pila y jabal inas quizás serían diferentes. Por lo que -también teóricamente- quizás habría entre ellos una cierta pauta diferencial que por el momento no conocemos, y que por lo tanto nos hace imposible todo tipo de discriminación si es que en realidad la hubo.

Como es bien sabido no es posible extrapolar sólidas conclusiones de este tipo atemporal de arma arrojadiza. No obstante la diferencia dimensional que caracteriza ambos grupos debe responder también a otras cuestiones tácticas y/o cuerpos de intervención asimismo distintos, bien de infantería, bien de caballería. En primer lugar, respecto a la longitud real del arma completa (moharra-hasta-regatón), lo que en la antigüedad respondería a vocablos diferentes como hoy lo es para «lanza» y «jabalina». En la actualidad como «lanza» las denominamos a la de mayor longitud, mientras que «jabalinas» serían las de más reducido tamaño como se nos muestran en la mayor parte de los hallazgos aquí presentados. Se corresponderían como tradicional mente se ha venido creyendo con el armamento ligero de los auxiliares de infantería tal y como se nos muestra uno de los relieves de $M$ ainz, o mismamente la pléyade de hallazgos en los castella. También en no pocas ocasiones se ha venido relacionando con la caballería (a quienes sería probable perteneciesen los hallazgos de Herrera) tal y como así nos lo indican, amén de otras tantas muestras arqueológicas (Kemkes y Scheuerbrandt, 1997: 37, fig. 30), las representaciones de jinetes en las estelas europeas de los diferentes partes del limes, 0 los pasajes literarios de Jenofonte (XII, 11) y J osefo (III, 92, 5). Quizás las lanzas de mayor tamaño pudieran también encontrarse relacionadas con ciertas tropas de infantería regular de ciudadanos, tal y como apreciamos en la estela de Publius F lauoleius Cordus soldado que fue de la legio XIIII Gemina (Gilbert, 2006: 30-33), que si bien es verdad, el tamaño de la lanza que porta dicho soldado es el de cualquier pilum.

\section{Pila}

De este tipo de arma arrojadiza que suponemos hoy fue tan característica de las alineaciones de infantería, tenemos una escasa y en ciertos aspectos dudosa documentación entre el volumen de objetos aquí estudiado (Quesada Sanz, 2008: 225-231). Es relativamente común que de los restos metál icos recuperados en las distintas ocupaciones militares que amortizaron el espacio donde hoy se encuentra Herrera de Pisuerga, hagan su aparición dos raros tipos de objetos formal mente repetitivos fabricados en cobre o aleación (quizás latón), que la arqueología militar romana aún no tiene convenientemente documentados ni identificados. El primero de ellos se trata de piezas en forma de aspa o cruz con los extremos de los brazos apuntados 0 redondeados (fig. 4,6), cóncavos los espacios entre ellos, y que normalmente fueron recortados a partir de una lámina ( $2 \mathrm{~mm}$ de grosor), aunque hay casos (como uno de los que aquí presentamos) en los que su espesor suele ser mayor (7 mm) (fig. 4,5). Cuando hacen su aparición estos supuestos «apliques» y que aún no han sido utilizados, su centro se encuentra taladrado mediante una perforación circular. En otros casos esta perforación central se encuentra realizada de forma esférica, como si se tratase del extremo de un pasador o remache disimuladamente pulido. Este pasador de sección circular se conserva en otro ejemplar de aplique cruciforme (fig. 4,7), cuya cabeza ha sido disimulada mediante pulido como decíamos anteriormente; ambas piezas provienen del nivel $\mathrm{V}$ de San M illán. 
Como atribución de este tipo de objetos como extremos más que decorativos de firme sustentación en «X», quedan los pasadores de los pila unidos de forma doble o triple a la madera y el metal. Este aserto viene de la mano de A chim Rost y Susanne W ilbers-R ost (U niversidad de Osnabrück) a partir de sus excavaciones en el conocido yacimiento de K alkriese, campo de batalla en el cual el año 9 d.C. fueron devastadas las tropas de P. Quintilio Varo por las poblaciones germanas, y cuya hipótesis han revelado en este mismo coloquio ${ }^{11}$. Como primera propuesta plausible apuntada por dichos investigadores aquí la recogemos, aunque en los pila que han conservado los extremos de los roblones de sujeción, estos son cuadrangulares 0 circulares (Connolly, 1997). L os ejemplares mostrados aparentemente son el ementos robustos al estilo del ejemplar $n=5$ de la figura $4^{12}$.

El segundo de los enigmáticos objetos son unas laminillas molduradas en toda la longitud de la superficie exterior, de entre 2-2'5 mm de grosor y entre 5-9 mm de anchura, dobladas en cuadrilátero con lados ligeramente oblicuos en sección y que por lo tanto forman lo que recordaría vagamente a un leve tronco de pirámide, y que enmarcan reducidos espacios (fig. 4,8); de los tres objetos que aquí mostramos dos proceden del nivel V de San M illán y el otro de El Castillo presentando las siguientes dimensiones: borde superior $21 \times 21 \mathrm{~mm}$ y borde inferior $23 \times 23 \mathrm{~mm}$; borde superior $23 \times 23 \mathrm{~mm}$ y borde inferior $25 \times 25 \mathrm{~mm}$; borde superior $17 \times 17 \mathrm{~mm}$ y borde inferior $19^{\prime} 5 \times 19^{\prime} 5 \mathrm{~mm}$. Son objetos que aparecen en todos los periodos campamentales de este yacimiento a lo largo del siglo I d.C. De entre la totalidad de hallazgos realizados, solo uno de entre todos ellos procede del área de San M illán y que aquí hemos presentado como primer ejemplo (fig. 4,8), desveló que las leves diferencias en el tamaño -milímetros- de los bordes superiores con respecto a los inferiores $( \pm 2 \mathrm{~mm}$ ) revelan el hecho de que tales leves desproporciones dimensionales respondían al motivo de que varios de estos objetos se sobreponían unos a otros encajando entre sí. De hecho los dos objetos antedichos de San M illán se encuentran superpuestos y levemente acoplados uno dentro de otro aproximadamente $1 \mathrm{~mm}$.

Podemos suponer que el primitivo y desconocido objeto en origen estaría compuesto por varios de estos pequeños «cuadradillos» superpuestos, hasta conseguir un pequeño tronco de pirámide de la longitud deseada. Buscando el símil formal para tal elemento, proponemos como hipótesis de trabajo que se tratase de zunchos que reforzaron la parte media de los pila, precisamente en la zona de unión entre metal y madera al ser esta última la zona más débil del arma. Es decir, la parte superior del hasta de madera, que se corresponde con zona media del arma (¿pirámide?); tope, bajo el cual se asía para su lanzamiento. Emplazado dicho engrosamiento, en el extremo distal del astil de madera, y donde precisamente encaja (y por sendos roblones metálicos queda firmemente unida) la placa inferior que caracteriza la moharra metálica de un pilum. Zona especialmente sensible a las roturas por cualquier tipo de flexión al tratarse de dos materias con diferente resistencia. Uno de los refuerzos troncopiramidales de los pila hallados en Oberanden (11-8 a.C.) posee unas dimensiones aproximadas de $25 \times 25$ mm (Bishop y Coulston, 1993: 66, 1a), aunque también los hay de mayores dimensiones y todos ellos en hierro. Los espacios que enmarcan estos zunchos cuadrados es en torno a los 20 $\mathrm{mm}$ de lado para los más pequeños, resultando espacio suficiente como para que a través de ellos pase la barra metálica (de sección cuadrada o circular) de un pilum, ya que dichas barras poseen una sección entre 8 y $20 \mathrm{~mm}$ de diámetro por ejemplo para los de sección circular halladas en Numancia (Luik, 2002: 227-229, A bb. 181, 182, 185). Por lo tanto esto evidencia

\footnotetext{
11 Ver dentro de este mismo volumen, la versión publicada de esta comunicación, infra, Rost \& Wilbers-Rost.

12 Un ejemplar en cruz con pasador de sección cuadrada realizado en al eación de cobre, fracturado, que vivamente nos recuerda los objetos aquí mostrados, procede de la necrópolis indígena de A rcóbriga (M onreal de A riza, Zaragoza) (L orrio y Sánchez de Prado, 2009: 230 no 353 y Fig. 116) que muestra una amplia cronología de uso entre los SS. IV-I a.C.
} 
un grosor mínimo en la madera en ese lugar, y de ahí la posible razón de ser de este elemento que aparentemente parece frágil, pero que se ve reforzado en su estructura física mediante los pliegues o costillas que actúan reforzando el fino grosor de la materia metálica. Algo parecido a lo que desde el punto de vista físico y de ingeniería industrial vemos actualmente en los recipientes cilíndricos de aluminio para el almacenamiento y consumo de bebidas carbónicas (Hosford y Duncan, 1994).

- A rtillería

Herrera de Pisuerga es un yacimiento en el que se han hallado escasas muestras de este tipo de armas fundamental mente de asedio. Tan solo contamos con una cabeza piramidal en hierro (67 mm de longitud) (fig. 4,10) para un dardo de catapulta accionado por torsión (García Díez y Sáez A bad, 2007: 455-460; Sáez A bad, 2005: 44 y ss.), con una característica poco usual en este modelo de cabeza de proyectil como es el poseer un sistema de sujeción por medio de un pequeño vástago circular ${ }^{13}$, en vez del tan abundante como también clásico enmangamiento cónico; procede del nivel $\mathrm{V}$ de San M illán.

También de este mismo yacimiento y nivel procede una robusta punta de flecha (48 $\mathrm{mm}$ de longitud) de triple aleta (fig. 4, 9). En base a este ejemplar apreciamos como estos modelos tan característicos del ejército y abundantes en época tardorepublicana han variado, tanto en tamaño como en forma. Habiendo aumentado sus dimensiones (aquellos con una longitud media entorno a los $\pm 25-55 \mathrm{~mm}$ ) y desaparecido los también triples extremos aguzados en forma de anzuelo (Peralta L abrador, 2007: 500). Este modelo de punta de flecha con mayor tamaño es característico del imperio ${ }^{14}$ y que pervive hasta los siglos V II-V III (García Jiménez, y Vivó i Codina, 2003: 178-179), siendo también característica su escasa aparición en número en cada yacimiento (D avies, 1977). Su presencia suel e estar ligada a tropas auxiliares que serían quienes las utilizasen ${ }^{15}$. Quizás un reducido grupo de especialistas, pero a todas luces no teniendo nada que ver con aquellos otrora contingentes de sagitarii.

\section{CONCLUSIONES}

De todo lo que anteriormente hemos expuesto, hay varias cuestiones que llaman la atención. En primer lugar la pequeña dimensión de los hallazgos, al go que resulta lógico debido a que se trata de hallazgos procedentes mayoritariamente de áreas de vertido. Estos vertederos son producto no solamente de los desechos arrojados de forma contínua en determinados lugares extra-muros como material inservible por parte de las tropas durante su devenir diario, sino también durante las diferentes remodelaciones del campamento. Estas grandes obras de transformación parece ser que tuvieron lugar en los castra legionis al menos en un par de ocasiones; la primera unos años antes del cambio de era, y la segunda durante el reinado de Tiberio (M orillo, Pérez e Illarregui, 2006: 317). 0 bien a posteriori una vez fue trasladada la

13 Un ejemplo de cómo permanecía insertada una cabeza de estas características en el extremo del cuerpo de un proyectil compuesto por dos tipos de maderas con diferente dureza, es uno de los hallazgos efectuados en Vindonissa y Haltern, ambas del siglo I d.C. (Iriarte, 2000: 67; Unz y Dechler-Erb, 1997: Taf. 22, 434).

14 Lo escaso de su aparición en época imperial es un dato que quizás nos esté poniendo en evidencia los cambios llevados a cabo en el ejército por A ugusto.

15 «... que arroje las flechas con destreza... » (Vegecio II, XIV, 7 - Sobre los escuadrones de la caballería legionaria- ), Compendio de técnica militar, edición y traducción a cargo de D. Paniagua A guilar, Ediciones Cátedra, M adrid (2006). 
legión al Rhin en torno al año 40 d.C., transformándose aquel ya antiguo campamento al gunos años después, con Nerón en el poder, en castellum de auxiliares con la llegada del ala Parthorum. L os sucesivos contingentes militares que ocuparon los cuarteles, también continuaron reutilizando aquellos vertederos.

En un lugar tan excepcional como fue la excavación de San M illán, la abundancia de material metálico muy diverso y de todo tipo en diferentes fases de fabricación y uso (en clara muestra diferencial respecto al resto de áreas de vertido, tanto en cantidad como en variedad), puso una vez más en evidencia la existencia -y como no podía ser de otra manera- de talleres (metalúrgicos en este caso concreto) -fabricae- (Bishop, 1985; Fernández Ibáñez, 1999: 251252). Q ue junto a la presencia de otros restos ya conocidos de otro tipo de producciones material es tales como la cerámica (común, sigillata, paredes finas y lucernas) y el hueso, demostró una vez más la coexistencia de un edificio específico, o grupo de estos. Ya fuese uno u otro, poseería grandes proporciones para albergar un volumen de producción y transformación de todo tipo de materias. De tal forma, que abasteciese tanto a la propia legión como no podría ser de otra manera en un ejército de ocupación, explotación, control territorial y social en un territorio lejano y hostil; y muy probablemente también a sus tropas de auxiliares. Es posible asimismo que estuviese ubicado cercano a la puerta Este del campamento (porta principalis sinistra).

Los objetos vistos nos hablan de una gran unidad militar compuesta por todo tipo de tropas, legionarios y auxiliares, ciudadanos romanos e indígenas, compuestos tanto por infantería como por caballería (Fernández Ibáñez, 2002a) y a su vez integrados en diversos regimientos especializados. Destacamentos formados por soldados que habían batallado previamente en otros frentes, y que portaban armas de la más diversa variedad como por otra parte fue siempre característico de y en la armada romana. Dichas armas, aunque su reconstrucción haya constituido un análisis cuasi-detectivesco debido mayoritariamente a su alta fragmentación, y en el cual la correcta limpieza de los objetos Ilevada a cabo por especialistas restauradores resulta una labor previa totalmente imprescindible, en su conjunto nos es posible detectar la presencia de un cambio a nivel formal, que enmascara otros de tipo histórico y estratégico ${ }^{16}$. L os objetos metál icos de la legio IIII M acedonica en Herrera de Pisuerga nos evidencian como esta fase se está Ilevando a cabo. Con la llegada de A ugusto a la púrpura imperial se produce también, entre otras muchas, una gran reforma en el ejército como es bien sabido, cambio que así mismo repercute en el armamento de manera progresiva, tornándose en un equipamiento más uniforme aunque nunca se consiga en su totalidad, y perdurando muchas de las armas en el tiempo. De esta manera vemos que desaparecen los regimientos de honderos y arqueros - 0 quedan reducidos al mínimo-, las dagas se transforman desapareciendo por ejemplo los modelos bidiscoidales, con el tiempo surgirá un nuevo tipo de coraza (lorica segmentata) que se complementará a las ya empleadas hasta entonces, se diseña un nuevo modelo de pilum, etc... Todo esto probablemente responda al desarrollo de unas y creación de otras nuevas tácticas en el combate, abandonando paulatinamente el esquema republicano.

16 Una gran reforma administrativa y militar llevada a efecto por A ugusto fue detectada en el campamento de Oberaden hacia el año 13 a.C. a partir del estudio de las monedas, y que parece constatarse también en Hispania (García y Bellido, 2004: 34, 49-50, 312). No obstante la transformación armamentística muy probablemente ya dio comienzo años antes. 


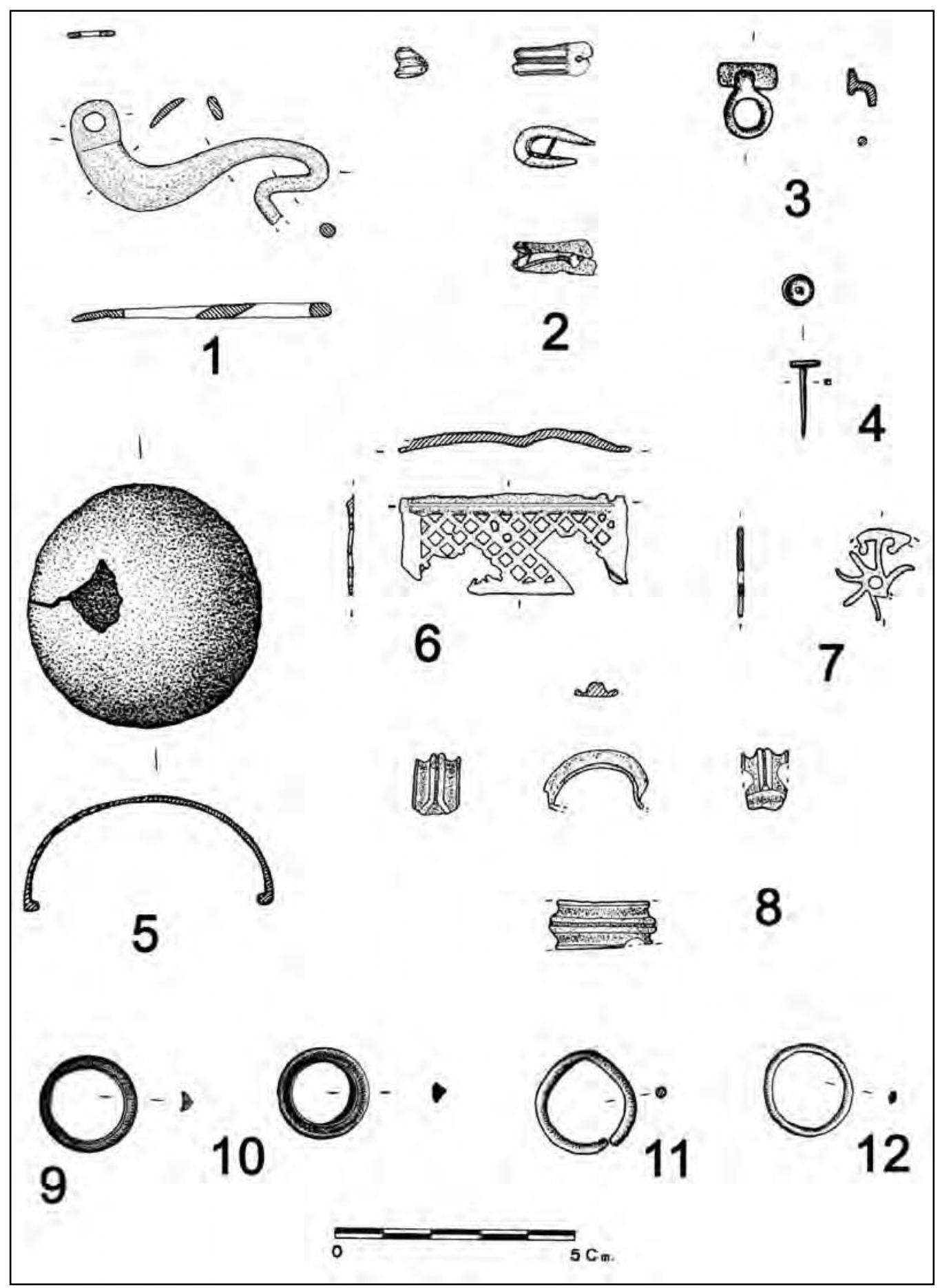

Figura 2. Herrera de Pisuerga. (1) A brochador/sustentador para solapas de lorica hamata; Col. Alba, M useo de Palencia. (2) Presilla para anilla de suspensión de vaina de daga. (3) B otón en «L» para la suspensión de dagas (?). (4) Clavillo para unión de placas de vaina de daga. (5) Semiesfera correspondiente al pomo de una espada. (6) Rejilla de vaina de espada. (7) Fragmento de rejilla de vaina de espada. (8) Presilla de zuncho de vaina de espada para la sustentación de la anilla. ( 9 a a12) A nillas. Todos los objetos se encuentran fabricados en aleación de cobre. 


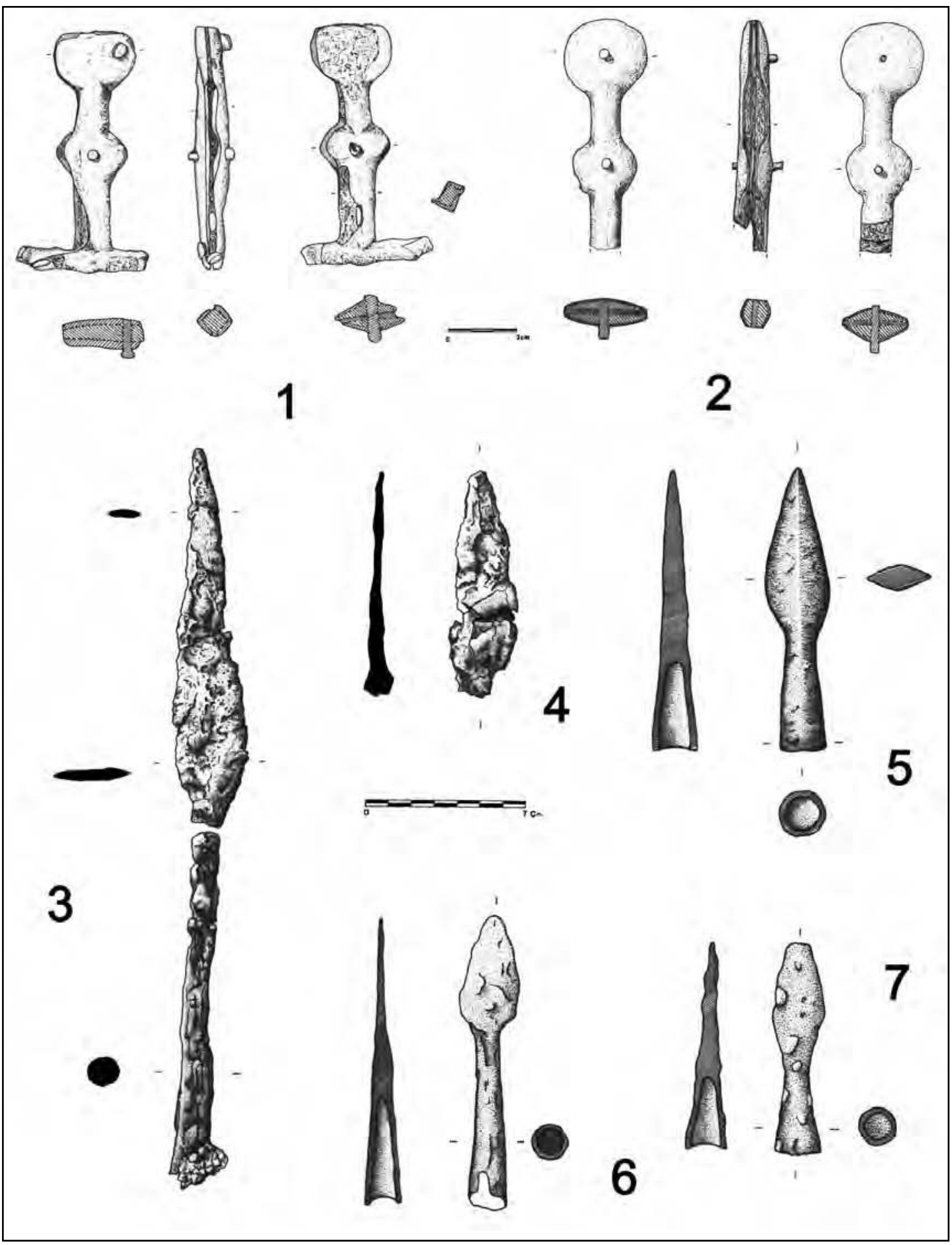

Figura 3. Herrera de Pisuerga. (1) Empuñadura de daga con pomo en «D»0 «semidisco». (2) Empuñadura bidiscoidal de daga. ( 3 a 7 ) M oharras de lanza. Todos los objetos se encuentran fabricados en hierro. 


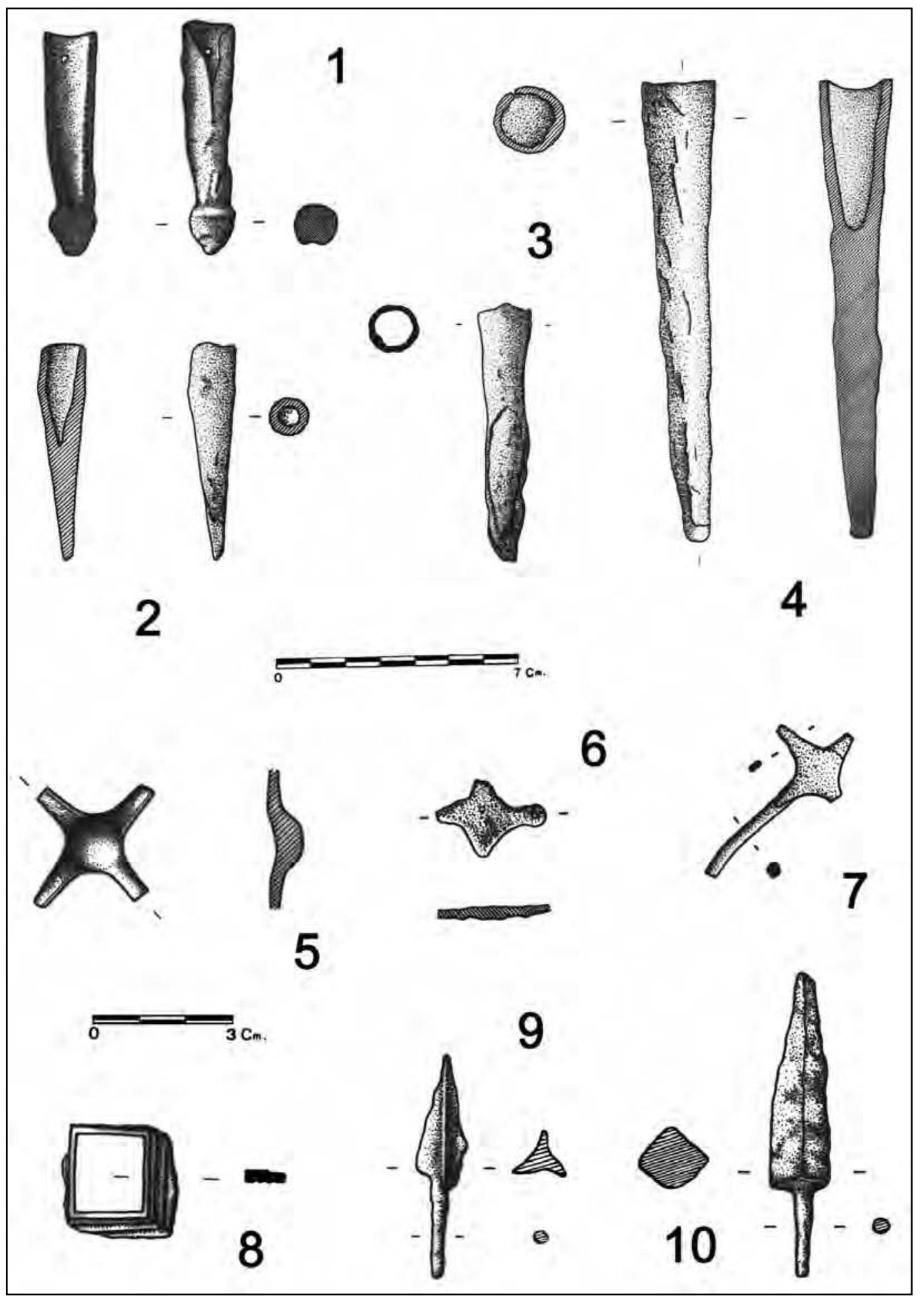

F igura 4. Herrera de Pisuerga. (1 a 4) Regatones. (5 a 7) Extremos de pasadores de sujeción de ahorras para pilum (?). (8) Fragmento de zuncho piramidal para la sujeción de moharra de pilum (?). (9) Punta de flecha de triple aleta. (10) Cabeza piramidal para dardo de catapulta (pilum catapultarium). L os objetos 1 a 4,9 y 10 se encuentran fabricados en hierro, el resto en aleación de cobre. 


\section{BIBLIOGRAFIA}

A Imagro-Gorbea, M. et alii (1996): «El poblado ibérico de El M olón». Revista de Arqueología, 181: 8-17.

Bishop, M . C. y Coulston, J. C. N . (1993): Roman military equipment. London.

Chew, H. (1991): «L es attaches de cotte de mailles», F. B reeck y H. Chew (eds.), M asques de F er. Un officier romain du temps de Caligula. Catalogue de l'exposition du M usée des antiquités nationales de Saint-Germain-en-Laye, 6 novembre 1991-4 février 1992. Paris: 38-44.

Connolly, P. (1997): «Pilum, Gladius and Pugio in the late Republic», M. Feugère (dir.), L'équipement militaire et l' armement de la République (IVe-|er $\mathrm{S}$. avant J .-C.). Journal of Roman M ilitary Equipment Studies, 8: 41-57.

Davies, J.L. (1977): «Roman A rrowheads from Dinorben and the Sagittarii of the Roman A rmy». Britannia, 8: 257-270.

Dechezlepretre, T. (2008): «Présence de militaria sur quelques oppida de I'est de la Gaule», M. Poux (ed.), Sur les Traces de César. Militaria tardo-républicains en contexte gaulois. Actes de la table ronde de Bibracte, 17 octobre 2002. Glux-en-Glenne: 93-102.

Desbat, A . y M aza, G. (2008): «M ilitaria de la moyenne vallée du R hône (Lyon, Valence, Vienne)», M. Poux (ed.), Sur les Traces de César. Militaria tardo-républicains en contexte gaulois. Actes de la table ronde de Bibracte, 17 octobre 2002. Glux-en-Glenne: 237-250.

Fernández Ibáñez, C. (1992): «El conocimiento de la antigua «Pisoraca» a través de los metales romanos». Papeles Herrerenses, I: 96-104.

Fernández Ibáñez, C. (1999a): «M etalistería y romanización en la antigua C antabria» J. M . I glesias y J. M uñiz (eds.): Regio Cantabrorum. Santander: 249-258.

Fernández Ibáñez, C. (1999b): «Placa de tahalí para la suspensión de las dagas en el ejército romano: entre la República y el Imperio. A propósito de un hallazgo en el campamento de la L egio IIII M acedónica (Herrera de Pisuerga, Palencia, España)», Estudios en Homenaje al Profesor D r. García Guinea. Sautuola, VI: 335-345.

Fernández Ibáñez, C. (2001): «Tropas auxiliares en Herrera de Pisuerga (Palencia). Nuevos datos», L. Hernández Guerra, J. M a . Solana Sáinz y L. Sagredo San Eustaquio (eds.), La Península Ibérica hace 2.000 años. Actas del ler Congreso Internacional de Historia Antigua (Valladolid, 23-25 de noviembre de 2000). Valladolid: 193-203.

Fernández Ibáñez, C. (2002): «M etalisteria bélica de la «L egio IIII M acedonica» procedente de su campamento en Herrera de Pisuerga (Palencia, España)», A. M orillo (ed.), A rqueología M ilitar Romana en Hispania. M adrid: 381-393.

Fernández Ibáñez, C. (2004a): «M etales romanos de Herrera de Pisuerga (Palencia). El yacimiento de «E| Cuartel - |». L os primeros asentamientos militares». Sautuola, X: 237-279.

Fernández I báñez, C. (2004b): «M etal istería militar romana en el norte de la Península Ibérica durante los periodos republicano y al toimperial», C. Fernández Ochoa y P. García (eds.), III er C oloquio Internacional de Arqueología en Gijón: Unidad y Diversidad en el Arco Atlántico en Época Romana. B.A.R. Int. Series, 1371. Oxford: 203-228.

Fernández Ibáñez, C. (2006a): «O bjetos metálicos del asentamiento militar de Herrera de Pisuerga (Palencia). Excavaciones de A. García y B ellido (1960-61)», M. B endala et alii (eds.), La Arqueología Clásica Peninsular ante el Tercer M ilenio. En el Centenario de A. García y Bellido (1903-1972). M adrid: 187-202.

Fernández Ibáñez, C. (2006b): «Post Vestigium Exercitus. M ilitaria romana en la región septentrional de la Península Ibérica durante la época A Itoimperial», A. M orillo (ed.), Arqueología militar romana en Hispania II: producción y abastecimiento en el ámbito militar. León: 257-308.

Fernández Ibáñez, C. (2007): «L a metalistería militar de H ispania en época altoimperial», C. Fernández Ibáñez (ed.), M etalistería Romana de Hispania. Sautuola, XIII: 403-426.

Fernández Ibáñez, C. (2008): «L as dagas del ejército altoimperial en Hispania». Gladius, XXVIII: 87175.

Fernández Ibáñez, C. (en prensa): «Objetos del pasado. A rqueología y conservación», Patrimonio y Restauración, Algo más que un Debate. Palencia. 
Fernández I báñez, C. y Pérez González, C. (1990): «Strigile romana procedente del yacimiento del «EI Cuartel» (Herrera de Pisuerga, Pal encia)», Actas del II0 Congreso de Historia de Palencia, Tomo I. Palencia: 431-441.

Feugere, M . (1993): Les armes des Romains, de la République à I'Antiquité tardive. Paris.

García Y Bellido, A . et alii (1962): Herrera de P isuerga. 1a Campaña. M adrid.

García Y B ellido, A . et alii (1970): Excavaciones y exploraciones arqueológicas en Cantabria. Madrid.

García-B ellido, M a . P. (2004): Las legiones hispánicas en Germania. M oneda y ejército. M adrid.

García Díez, F. y Sáez A bad, R. (2007): «La artillería de la Hispania romana», C. Fernández Ibáñez (ed.) M etalistería de la Hispania Romana. Sautuola, XIII: 445-464.

García Jiménez, G. y Vivó I Codina, D. (2003): «Sant Julià de Ramis y Puig Rom: dos ejemplos de yacimientos con armamento y reequipamiento militar visigodo en el noroeste peninsular», Gladius, XXIII: 161-190.

Gilbert, F. (2006): Légionnaires et auxiliaires sous le H aut-E mpire Romain. Paris.

Hosford, W. F. y Duncan, J. L. (1994): «L atas de refrescos». Investigación y Ciencia, 218: 20-26.

Iriarte, A. (2000): «Pseudo-Heron's cheiroballistra a(nother) reconstruction, I.: Theoretics». J ournal of Roman Military Equipment Studies, 11: 47-75.

James, S. (2004): Excavations at Dura-Europos 1928-1937. The Arms and Armour and other Military Equipment. L ondon.

K avanagh de Prado, E. (2008): «El puñal bidiscoidal peninsular: tipología y relación con el puñal militar romano (pugio)». Gladius, XXVIII: 5-85.

Kemkes, M. y Scheuerbrandt, J. (1997): Zwischem Patroville und Parade. Die Römische Reiterei am Limes. Stuttgart.

Lorrio, A. J. y Sánchez De Prado, Mạ. D. (2009): La necrópolis de Arcóbriga. Caesaraugusta, 80: $1-565$.

Luik, M . (2002): Die F unde aus den Römischen Lagern um Numantia im Römisch-Germanischen Zentralmuseum. Mainz.

M artínez Santa-Olalla, J. (1933): Excavaciones en la necrópolis visigoda de Herrera de Pisuerga (Palencia). M adrid.

M orillo Cerdán, A . (1999): Lucernas romanas en la región septentrional de la Península Ibérica. M ontagnac.

M orillo Cerdán, A . (2000): «L a legio IIII M acedonica en la península I bérica. El campamento de Herrera de Pisuerga (Palencia)», Y. Le B ohec (ed.), Les Légions de Rome sous le H aut-E mpire. Vol. II. Lyon: 609-624.

M orillo Cerdán, A . y Fernández Ibáñez, C. (2001-02): «Un aplique decorativo con inscripción militar procedente de Herrera de Pisuerga (Palencia, España)». J ournal of Roman Military Equipment Studies, 12-13: 47-51.

M orillo Cerdán, Á . y Gómez Barreiro, M. (2006): «Circulación monetaria en Herrera de Pisuerga (Palencia)», M a. P. García-B ellido (ed.), Los Campamentos Romanos en Hispania (27 a.C.-192 d.C.). Vol. I. M adrid: 338-438.

M orillo Cerdán, Á ., Pérez González, C. e Illarregui, E. (2006): «Herrera de Pisuerga (Pal encia). Introducción histórica y arqueológica. Los asentamientos militares», M ạ. P. García-Bellido (ed.), Los Campamentos Romanos en Hispania (27 a.C.-192 d.C.). Vol. I. M adrid: 305-323.

Oldenstein, J. (1976-77): «Zus A urrüstung römischer A uxiliareinheiten», Bericht der Römisch-G ermanischen K ommission, 75: 49-186.

Peral ta Labrador, E. (2007): «E qui pamiento militar romano de la conquista de la antigua Cantabria», C. Fernández Ibáñez (ed.), M etalistería de la Hispania Romana. Sautuola, X III: 493-525.

Pérez González, C. (1989): Cerámica romana de Herrera de Pisuerga (Palencia, España). La terra sigillata. Santiago de Chile.

Pérez González, C. y A rroyo Rodríguez, L. A . (2003): Herrera de Pisuerga: aproximación histórica. Palencia.

Pernet, L., Poux, M . y Teegen, W-R . (2008): «M ilitaria gaulois et romains sur I'oppidum de Bibracte, M ont B euvray (Nièvre)», M. Poux (ed.), Sur les Traces de César. Militaria tardo-républicains en contexte gaulois. Actes de la table ronde de Bibracte, 17 octobre 2002. Glux-en-Glenne: 103-139. 
Poux, M. (2008): «L'empreinte du militaire tardo-républicain dans les faciès mobiliers de La Tène finale. Caractérisation, chronologie et diffusion de ses principaux marqueurs». M. Poux (ed.): Sur les Traces de César. Militaria tardo-républicains en contexte gaulois. Actes de la table ronde de Bibracte, 17 octobre 2002. Glux-en-Glenne: 299-432.

Poux, M ., Feugere, M y Demierre, M. (2008): «A utour de Gergovie. Découvertes anciennes et récentes», M . Poux (ed.), Sur les Traces de C ésar. M ilitaria tardo-républicains en contexte gaulois. Actes de la table ronde de Bibracte, 17 octobre 2002. Glux-en-Glenne: 203-223.

Quesada Sanz, F. (2008): Armas de G recia y Roma forjaron la historia de Antigüedad clásica. M adrid. Sáez A bad, R. (2005): Artillería y poliorcética en el mundo grecorromano. M adrid, CSIC.

Unz, Ch. y Deschler-Erb, E. (1997): Katalog der militaria aus Vindonissa. Brugg.

Wild, J. P. (1970): «Button-and-L oop Fasteners in the Roman Provinces». Britannia, 1: 137-155.

Recibido: $25 / 01 / 2010$

A ceptado: $12 / 04 / 2010$ 